\title{
MANEJO DE LA ANSIEDAD EN EL PACIENTE PEDIÁTRICO ONCO- LÓGICO Y SU CUIDADOR DURANTE LA HOSPITALIZACIÓN A TRAVÉS DE MUSICOTERAPIA
}

\author{
ANXIETY MANAGEMENT IN PEDIATRIC CANCER PATIENT AND THEIR \\ CAREGIVER DURING HOSPITALIZATION THROUGH MUSIC THERAPY
}

María del Consuelo Cabral-Gallo', Alma Olga Delgadillo-Hernández', Esther Margarita Flores-Herrera ${ }^{2}$ y Fernando Antonio Sánchez-Zubieta ${ }^{1}$

Área de Psicooncología. Instituto de Investigación del Cáncer en la Infancia y Adolescencia. Departamento
de Reproducción Humana y Crecimiento Infantil. Centro Universitario de Ciencias de la Salud. Universidad
de Guadalajara. México.
Facultad de Psicología. Universidad de Guadalajara. México.

Resumen

Objetivo: Evaluar la eficacia de la musicoterapia para el manejo de ansiedad durante el periodo de hospitalización en el paciente pediátrico oncológico y su cuidador.

Método: Se realizó medición antes y después de una única sesión musical y se comparó resultado de la evaluación de ansiedad con grupo testigo. Participaron 112 pacientes pediátricos oncológicos y 128 cuidadores. Se aplicó la Escala de Ansiedad Manifiesta (CMAS-R; Reynolds y Richmond, 1997) en los pacientes y la Escala Hamilton (HAS; Hamilton, 1959) en el cuidador. El análisis estadístico se realizó por medio del programa SPSS Versión 20.

Resultados: No se detectó cambio significativo en el grupo de pacientes, en quienes el tamaño del efecto se considera mínimo, mientras que en los cuidadores se presentó resultado significativo en 12 de las 13 dimensiones de ansiedad evaluadas, con un tamaño del efecto mediano.

Conclusiones: El uso de musicoterapia ayuda a disminuir el nivel de ansiedad en el cuidador, mientras que el resultado obtenido en
Abstract

Objective: Evaluate the efficacy of music therapy for the management of anxiety during the period of hospitalization in pediatric cancer patient and caregiver.

Method: Measurement was performed before and after a single music session and compared result of the evaluation of anxiety with control group. 112 patients and 128 caregivers collaborated. The level of anxiety was assessed using Manifest Anxiety Scale (CMAS-R, Reynolds \& Richmond, 1997) applied to patients and the Hamilton Scale (HAS; Hamilton, 1959) for caregivers. Statistical analysis was performed using SPSS version 20 program.

Results: No significant change was detected in the group of patients in whom the size of the effect is minimal, whereas caregivers provided significant results in 12 of the 13 dimensions of anxiety assessed with a medium effect size.

Conclusions: The use of music therapy helps to decrease the anxiety level significantly in caregiver, while the results obtained from

\section{Correspondencia:}


el paciente pediátrico oncológico no sostiene la idea generalizada del beneficio de la musicoterapia para el manejo de ansiedad.

Palabras Clave: Ansiedad, musicoterapia, cáncer infantil, hospitalización. the cancer patients did not support the idea of the benefits of releving anxiety.

Key words: Anxiety, music therapy, childhood cancer, hospitalization.

\section{INTRODUCCIÓN}

La vivencia de ansiedad en los niños y adolescentes que padecen cáncer se despierta de forma repetida debido a que son sometidos a hospitalizaciones frecuentes durante el periodo de tratamiento, es un acontecimiento que altera su proceso normal de actividades, pues implica estar expuestos a la serie de consultas, exámenes y rutinas propias de los tratamientos médicos, estar en contacto con varios profesionales de la salud, se enfrentan además al miedo por los procedimientos dolorosos, a las ideas preconcebidas con relación al cáncer y a la posibilidad de muerte. Es posible dilucidar que el paciente pediátrico oncológico afronta cambios en todas las áreas: física, emocional, psicológica, social, familiar y escolar ya que en gran medida, el contexto hospitalario irrumpe en su etapa de desarrollo, afecta sus relaciones interpersonales debido a que les aísla de su vida cotidiana y de sus redes de apoyo más próximas. Valdés \& Flores ${ }^{(1)}$ han destacado cinco categorías en las que se puede clasificar la amenaza para el niño ante la hospitalización: separación de los padres, los extraños, la pérdida de control, autonomía y competencia; la experiencia de dolor y la intervención quirúrgica así como la anestesia. Por su parte, May ${ }^{(2)}$ plantea que la ansiedad puede comprenderse como la reacción básica de los seres humanos hacia un peligro sobre su existencia, o hacia algún valor que él/ella identifique en su existencia. Es así que la ansiedad consti- tuye una consideración particular y es un foco importante de atención a la hora de plantear la intervención psicooncológica.

Por otro lado, el cuidador principal del paciente durante el periodo de hospitalización generalmente es alguno de los padres, quien vive este proceso en relación directa a la angustia, afecta su rutina diaria a nivel individual y en el sistema familiar, pues además vigilan la integridad emocional, psicológica y física del resto de los miembros de la familia. Los cuidadores presentan altos niveles de estrés, angustia y miedo que en ocasiones desencadena estados de crisis de ansiedad. En la óptica de éste estado emocional, se sustenta el hecho que en repetidas ocasiones existe transferencia entre cuidador-paciente que incrementa y mantiene estados altos de ansiedad en ambos durante el periodo de hospitalización. Esta misma transferencia puede darse del paciente hacia el cuidador, lo cual puede llegar a ser alarmante, ya que es muy probable que repitan episodios de ansiedad durante el tiempo de tratamiento, con riesgo sobreañadido de generar mayor conflicto tanto emocional como psicológico que puede incluso reflejarse en el incremento de la tasa de abandono a tratamiento.

Así, al considerar estos aspectos relacionados con la ansiedad en el niño y adolescente con cáncer y en su cuidador durante la hospitalización, se parte del supuesto que la musicoterapia es una opción favorable que ayuda a disminuir el nivel y frecuencia de los episodios de ansiedad; por lo que se busca en la bibliografía todos aquellos estudios que han aportado al 
tema. Para el niño hospitalizado, la musicoterapia puede ser una alternativa de comunicación, ya que ofrece la oportunidad de expresar sus emociones sin necesidad de ponerle palabras, es una de las mejores formas de tratamiento para los niños que padecen serias enfermedades y es una opción terapéutica al proporcionar bienestar, autoconfianza, relajación y al ayudar a la liberación de agentes químicos que regulan los estados de ánimo depresivos o los comportamientos agresivos ${ }^{(3,4)}$. Las investigaciones efectuadas con Musicoterapia en el campo de la oncología muestran resultados muy satisfactorios en la disminución de la ansiedad, estrés y depresión, proporcionando ayuda, soporte emocional y consuelo, que facilitan un mejor afrontamiento psicológico y espiritual ante la enfermedad y la posible muerte ${ }^{(5)}$. Algunos expertos en el campo de la musicoterapia recomiendan una variedad de música clásica para tratar de mejorar trastornos como la ansiedad, depresión e insomnio, entre otros trastornos que son experimentados frecuentemente por los niños en su estancia en el hospital ${ }^{(4)}$.

En búsqueda de contar con nuevos recursos para el manejo de ansiedad en el paciente con cáncer y su cuidador, en el Área de Psicología del Servicio de Hemato-Oncología Pediátrica del Hospital Civil de Guadalajara "Dr. Juan I. Menchaca" (HCGJIM), se decide llevar a cabo la presente investigación bajo el conocimiento previo de la experiencia de la aplicación de musicoterapia en pacientes oncológicos en países como Australia, Canadá Europa, Japón, Estados Unidos y Reino Unido(6), donde se ha incorporado la musicoterapia por ser un recurso que genera además del beneficio al oyente, la suficiente evidencia que permite describir, explicar y predecir el fenómeno de manera consistente. La musicoterapia, en sus múltiples campos de aplicación y, especialmente en el ámbito médico, está avalada por la Historia del uso terapéutico de la música y los aportes de la Psico-neuro-endocrino-inmunología $^{(7)}$. La Nacional Association for Music Therapy (NAMT) la define como el uso de la música en la consecución de objetivos terapéuticos, contribuyendo al mantenimiento e incremento de la salud física y mental. De igual forma, Ortiz ${ }^{(8)}$ considera que la musicoterapia en el ámbito hospitalario ayuda a reducir la tensión, brinda una sensación confortable en el niño, facilita la expresión de sentimientos y promueve movimiento e imitaciones.

De esta manera, en un intento por ayudar al paciente pediátrico oncológico y a su cuidador al manejo de la ansiedad durante el periodo de hospitalización, se pretende el uso de la musicoterapia como elemento complementario en la intervención psicooncológica, pues al conocer los resultados favorables en las investigaciones analizadas y siendo nuestro objetivo principal la disminución en los niveles de ansiedad, se buscó que la presente investigación generase nuevos aportes bajo la siguiente particularidad metodológica: evaluar el nivel de ansiedad de forma directa en el paciente y cuidador antes y después de la sesión musical, y comparar el resultado obtenido con un grupo testigo bajo los mismos criterios de inclusión y medición de ansiedad, aunque sin el estímulo musical. A partir de lo anterior, los resultados que se presentan a continuación permitirán determinar si la aplicación de la musicoterapia incide de forma positiva en la disminución de los niveles de ansiedad, con el objetivo de evaluar la efectividad de la música como herramienta de apoyo durante el tratamiento en hospitalización.

\section{MÉTODO}

\section{Participantes}

La unidad de análisis fue el paciente oncológico y su cuidador. En el caso del 
paciente se consideraron los siguientes criterios de inclusión: 1) tratamiento activo con quimioterapia; 2) edad entre seis y 18 años en ambos sexos; 3) estar hospitalizado al momento de la medición; 4) desear colaborar en el estudio. Se empleó formula estadística para población finita al Universo total de pacientes del Servicio de Hemato-oncología pediátrica (HCGJIM) con los criterios antes señalados. El número de muestra requerido para ser representativo a ésta población fue de 112 individuos, los cuales se dividieron entre grupo intervención y testigo con 56 participantes cada uno. En el caso de los cuidadores, el grupo intervención se conformó por 64 participantes, muestra que se homologó al grupo testigo, con un total de 128 participantes. Los criterios de inclusión fueron: 1) ser cuidador principal del paciente durante el periodo de hospitalización y 2) participación voluntaria.

El reclutamiento de participantes se realizó en el área de hospitalización del Servicio de hemato-oncología pediátrica (HCJIM) en las cuatro habitaciones que albergan 6 camas cada una. La promoción fue a través de la invitación directa a participar en la investigación tanto a pacientes como a sus cuidadores y se obtuvo consentimiento informado. En el grupo de pacientes con intervención participaron 32 niños y 24 niñas, mientras que en el grupo testigo fueron 29 niños y 27 niñas. En el caso de los colaboradores (papás), participaron en el grupo intervención seis hombres y 58 mujeres, mientras que en el grupo testigo fueron 18 hombres y 46 mujeres.

\section{Instrumentos}

La ansiedad en el paciente se evaluó con la Escala de Ansiedad Manifiesta en niñosrevisada (C-MAS-R) de Reynolds y Richmond desarrollada en $1997^{(9)}$, la cual consta 37 ítems correspondientes a cinco dimensiones:
Ansiedad Total; Ansiedad fisiológica; Inquietud/hipersensibilidad; Preocupación social y Mentira. Cada una con coeficiente elevado de confiabilidad valorado de acuerdo a edad, sexo y grupo étnico. El instrumento cuenta además con análisis de validez desde tres componentes: contenido, constructo y criterio. La prueba está diseñada para aplicarse de forma individual o grupal en niños de seis a 18 años y para su evaluación, la suma de respuestas positivas determina el nivel de ansiedad.

La medición del nivel de ansiedad en el colaborador se llevó a cabo a través de la Escala de Ansiedad Hamilton (HAS), prueba desarrollada por Max Hamilton en 1959, se destaca por sus propiedades psicométricas pues cuenta con amplia validez y confiabilidad en relación con otros instrumentos de su tipo. La escala consta de 13 ítems mediante los cuales se explora ansiedad, tensión, síntomas neurovegetativos y somáticos, logrando obtener una valoración cuantitativa en cada participante. Para esta investigación se empleó la versión española del Instrumento desarrollada por Carrobles et al. ${ }^{(10)}$.

Se consideró oportuno incluir el análisis de confiabilidad respecto a los instrumentos de medición CMAS-R y HAS con la finalidad de garantizar que los resultados son confiables. Al correr la prueba alfa de Cronbach en los pacientes del grupo con intervención se obtiene puntuación de 0,898 mientras que en grupo testigo fue 0,916 . En la interpretación de confiabilidad en los cuidadores del grupo con intervención señala puntuación de 0,910 y en el grupo testigo 0,932, por lo que ambos grupos cuentan con alta confiabilidad.

\section{Procedimiento}

Esta investigación se ajusta a un diseño cuasi-experimental, de tipo analítico, transversal, donde la musicoterapia se sometió a prueba como recurso para ayudar 
a disminuir los niveles de ansiedad. Para tal efecto, se desarrolló un estudio controlado no aleatorio con grupo intervención y testigo, este diseño ayudó a conocer si la musicoterapia influye en el nivel de ansiedad del participante al momento de la medición. El trabajo de campo en ambos grupos se llevó a cabo dos veces por semana con una duración aproximada de 2 horas con inicio puntual a las 9:00 a.m. en sólo una de las habitaciones del Servicio, eligiendo aquella en la que por edad de los pacientes se pudiera captar mayor número de participantes. Se comenzó con la invitación a participar en el estudio y en caso afirmativo se firmó consentimiento informado. Los instrumentos fueron aplicados por psicólogas adscritas al Servicio de Hemato-oncología pediátrica, mientras que la sesión musical fue aplicada y desarrollada por una psicóloga con licenciatura en música y especialidad en violín. Primero se desarrolló la medición con el grupo intervención hasta completar el número de muestra requerido y después se desarrolló la medición para el grupo testigo. La medición de ansiedad en el grupo con intervención se llevó a cabo entre los meses de Junio a Agosto del 2012, tiempo en el que se completó la mitad de la muestra requerida según la formula estadística para población finita al universo total de pacientes del Servicio. En el caso del grupo testigo, la medición de ansiedad comprendió los meses de Enero a Mayo del 2013.

En el grupo con intervención se aplicó el instrumento antes y después de la sesión musical (véase anexo), la cual se conformó por dos melodías de música clásica, dos de música folclórica, una instrumental y una de música medieval. La selección de las obras musicales cumplió con un orden, tiempo de ejecución y requisitos específicos para lograr el objetivo del presente estudio. Los participantes escucharon una única sesión musical y en total se desarrollaron 13 sesiones musicales diferentes, pues fueron las requeridas para completar el número total de la muestra para obtener una medición representativa en la población de pacientes del servicio de Hemato-oncología pediátrica. Durante la medición, la habitación permaneció cerrada para evitar interrupciones, el personal de enfermería llevó a cabo la administración de medicamentos conforme lo requerido en el paciente, por lo que no se irrumpió en ningún momento las indicaciones médicas. En el caso del grupo testigo, la invitación y desarrollo de la medición consta de las mismas fases que el grupo con intervención aunque sin la sesión musical. Durante la pre y post evaluación se desarrollaron las actividades normales en la habitación, en este caso, además de la administración de medicamentos se llevaron a cabo las actividades de terapia lúdica que se desarrollan entre semana en todas las habitaciones del Servicio.

Finalmente, el análisis estadístico se realizó a través del programa SPSS versión .20 para Windows. Se utilizó media y desviación estándar para describir las variables clínicas. La prueba de $t$ de Student se empleó para el análisis de las diferencias entre puntuaciones estadísticamente significativas de las variables. Se compararon las puntuaciones entre grupo intervención y testigo. El nivel de significación estadística se fijó en $p<0,05$.

\section{RESULTADOS}

En esta sección se presentan primero el resultado con los 112 pacientes pediátricos con diagnóstico oncológico que cursaron por periodo de hospitalización durante su tratamiento; de forma consecutiva se muestra el resultado de los 128 cuidadores participantes. Se despliegan tablas y gráficas para una fácil representación de los resultados con significancia estadística. 


\section{Pacientes}

Participaron 61 pacientes del sexo masculino y 51 del sexo femenino, las edades oscilaron entre los seis y 18 años siendo éste uno de los criterios de inclusión. La edad de los pacientes del grupo intervención presentó una media de 11,14 años, con DT de 3,6; la edad más frecuente fue 12 años y la menos frecuente fueron los 10 años. El grupo testigo de pacientes presentó media en la edad de 11,16 con DT de 3,73 ; el rango de edad con mayor frecuencia fue 12 años y la de menor frecuencia fue 10 años. La prueba $t$ de Student para comparación de edad entre grupos evidencia que no hay diferencia significativa, lo que permite que sean comparables respecto a esta variable.

La evaluación de ansiedad mediante $C M A S-R$ en el grupo intervención evidenció una media de 12,71 en la puntuación natural de Ansiedad Total antes de la sesión musical, mientras que en la post evaluación se obtuvo una media de 11,95. En el caso del grupo testigo, la pre evaluación muestra una media de 13,89, mientras que en la post evaluación la media fue de
13,21 (ver tabla 1). Para poder afirmar que hay diferencia en el nivel de ansiedad al emplear musicoterapia, posterior al contraste de medias y a la valoración de resultados con significancia estadística mediante la $t$ de student, se llevó a cabo un análisis complementario para conocer el tamaño del efecto, considerando así la magnitud del resultado o si la diferencia en la medida va de pequeña a grande, para de esta manera, plantear la relevancia de la diferencia encontrada. En este caso la pre evaluación muestra tamaño del efecto de 0,20 (pequeño), mientras que en la post evaluación se obtiene 19 que por igual es efecto pequeño.

Con relación a las otras cuatro dimensiones valoradas a través de CMAS-R, se identificó que el género masculino no presenta resultado significativo en ninguno de los grupos, mientras que el femenino en el grupo intervención obtiene resultado significativo de 0,004 en la dimensión Ansiedad Fisiológica y 0,028 en la dimensión Hipersensibilidad, mientras que el grupo testigo refleja 0,043 en Ansiedad Fisiológica, siendo la única dimensión con significación estadística aún sin el estímulo musical (figura 1).

\section{Tabla 1. Comparación entre puntaje de Ansiedad Total Pre y post (intergrupo) en} ambos grupos.

\begin{tabular}{|c|c|c|c|c|c|c|c|c|}
\hline Género & Medición & Grupo & Media & ds & $\begin{array}{c}\text { Error típ. } \\
\text { de la } \\
\text { media }\end{array}$ & $\mathbf{N}$ & $\begin{array}{c}\text { Delta } \\
\text { de } \\
\text { Cohen }\end{array}$ & $\begin{array}{c}\text { ds delta } \\
\text { de } \\
\text { Cohen }\end{array}$ \\
\hline \multirow{4}{*}{ Masculino } & Pre & Intervención & 12,25 & 5,91 & 1,05 & 32 & & \\
\hline & & Testigo & 12,41 & 6,29 & 1,17 & 29 & & \\
\hline & Post & Intervención & 12,19 & 6,68 & 1,18 & 32 & & \\
\hline & & Testigo & 12,55 & 7,31 & 1,36 & 29 & & \\
\hline \multirow{4}{*}{ Femenino } & Pre & Intervención & 13,33 & 4,68 & 0,95 & 24 & & \\
\hline & & Testigo & 15,48 & 5,98 & 1,15 & 27 & & \\
\hline & Post & Intervención & 11,63 & 5,55 & 1,13 & 24 & & \\
\hline & & Testigo & 13,93 & 6,44 & 1,24 & 27 & & \\
\hline \multirow{4}{*}{ Totales } & Pre & Intervención & 12,71 & 5,40 & 0,72 & 56 & 0,20 & 0,1 \\
\hline & & Testigo & 13,89 & 6,28 & 0,84 & & & \\
\hline & Post & Intervención & 11,95 & 6,18 & 0,83 & 56 & 0,19 & 0,09 \\
\hline & & Testigo & 13,21 & 6,88 & 0,92 & & & \\
\hline
\end{tabular}




\section{Figura 1. Dimensiones pareadas intragrupo por género en ambos grupos en el CMAS-R}

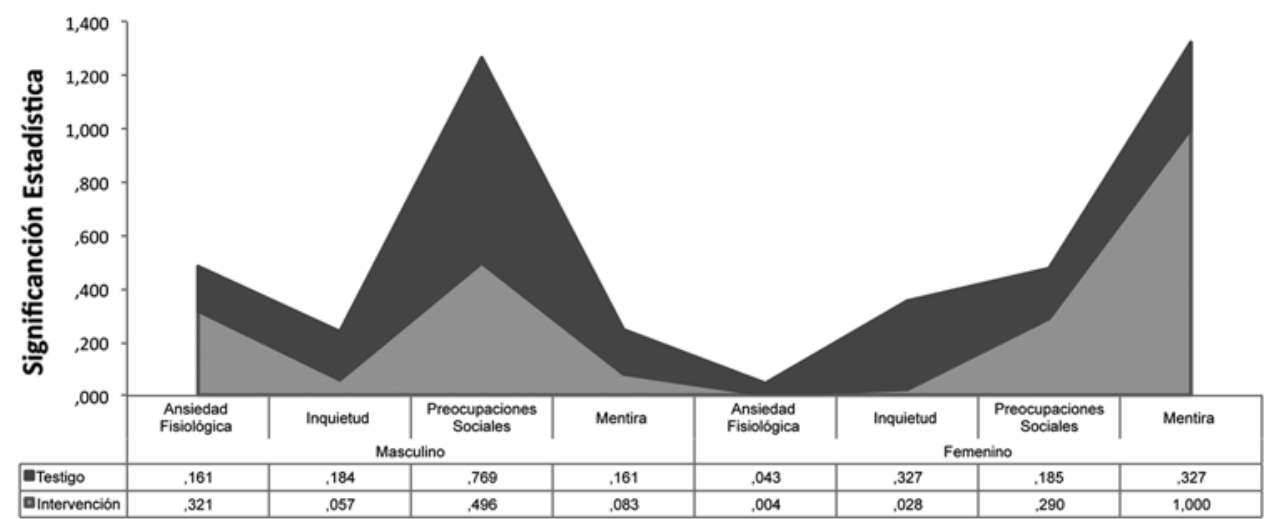

\section{Cuidadores}

Participaron 24 hombres y 104 mujeres. La media de edad calculada en el grupo con intervención fue 35,39 años, la edad más frecuente fue 37 años y la menos frecuente los 33 años; en el grupo testigo la media de edad fue de 38,38. La de mayor frecuencia fueron los 40 años y la de menor frecuencia 36 años. Lo anterior refleja que no hay diferencia significativa en ambos grupos respecto a esta variable según la $t$ de student, por lo que sus resultados pueden ser comparados entre sí.

El nivel de Ansiedad Total en la pre medición según el puntaje obtenido a través de la Escala HAS, refleja en el grupo con intervención una media de 1,69 mientras que el grupo testigo la media fue de 1,52 en tanto la ds en ambos es de 1,00. De esta forma, se observa que el tamaño del efecto es 0,16 por lo que no logra ser significativo al no alcanzar el mínimo del efecto esperado. En tanto, la post medición del grupo con intervención obtiene una $d$ de Cohen de 0,34 por tanto es efecto moderado tabla 2).

Dado que el tamaño del efecto fue moderado en el grupo intervención de cuidadores, se consideró relevante incluir la medición en cada una de las dimensiones de la Escala HAS, para ello se contrastaron las medias intergrupo y se comparó

\section{Tabla 2. Comparación entre puntaje de Ansiedad Total pre y post (integrupo) en los cuidadores}

\begin{tabular}{llllllll}
\hline Dimensión & Medición & Grupo & Media & ds & $\begin{array}{c}\text { Error } \\
\text { típ. de la } \\
\text { media }\end{array}$ & $\begin{array}{c}\text { Delta de } \\
\text { Cohen }\end{array}$ & $\begin{array}{c}\text { ds delta de } \\
\text { Cohen }\end{array}$ \\
\hline & Pre & Intervención & 1,69 & 1,00 & 0,126 & & \\
\multirow{2}{*}{$\begin{array}{c}\text { Ansiedad } \\
\text { Total }\end{array}$} & & Testigo & 1,52 & 1,00 & 0,126 & 0,16 & 0,98 \\
& Posterior & Intervención & 1,08 & 0,981 & 0,123 & & \\
& & Testigo & 1,45 & 1,14 & 0,142 & 0,34 & 0,17 \\
\hline
\end{tabular}


resultado con el grupo testigo. Se detectaron cambios significativos en 12 de las 13 dimensiones en el grupo intervención, mientras que en el grupo testigo fueron sólo 2 dimensiones con significancia estadística. De igual forma, se llevó a cabo el análisis del tamaño del efecto en cada una de las dimensiones, las cuales se midieron a través de la $d$ de Cohen (tabla 3 ).
Síntomas Somáticos Generales, Síntomas Respiratorios y Genitourinarios. Las dimensiones con tamaño moderado $(0,20$ a 0,50) son seis: Miedo, Insomnio, Funciones Intelectuales, Síntomas Somáticos, Síntomas Cardiovasculares y del SNC Autónomo. Un efecto grande (más de 0,50 ) se obtuvo en dos dimensiones: Humor Ansioso y Tensión.

\section{Tabla 3. Dimensiones Hamilton Pareadas y Deltas en Grupo Colaboradores}

\begin{tabular}{|c|c|c|c|c|c|c|c|c|c|c|}
\hline \multirow[b]{2}{*}{ Dimensión Hamilton } & \multicolumn{4}{|c|}{ Grupo Intervención } & \multicolumn{4}{|c|}{ Grupo Testigo } & \multirow[b]{2}{*}{$\begin{array}{l}\mathrm{d} d \mathrm{de} \\
\text { Cohen }\end{array}$} & \multirow[b]{2}{*}{$\begin{array}{c}\text { ds } \\
\text { Cohen }\end{array}$} \\
\hline & Media & $\begin{array}{c}\text { ds } \\
\text { típica }\end{array}$ & $\begin{array}{c}\text { Error típ } \\
\text { de la } \\
\text { media }\end{array}$ & Sig. & Media & $\begin{array}{c}\text { ds } \\
\text { típica }\end{array}$ & $\begin{array}{l}\text { Error } \\
\text { típ. } \\
\text { de la } \\
\text { media }\end{array}$ & Sig. & & \\
\hline Humor Ansioso Pre-Post & 0,766 & 10,02 & 0,127 & 0,000 & 0,219 & 0,70 & 0,088 & 0,015 & 0,625 & 0,289 \\
\hline Tensión Pre-Post & 0,781 & 0,88 & 0,110 & 0,000 & 0,094 & 10,26 & 0,157 & 0,553 & 0,633 & 0,302 \\
\hline Miedo Pre-Post & 0,500 & 0,89 & 0,111 & 0,000 & 0,141 & 0,87 & 0,109 & 0,201 & 0,408 & 0,1997 \\
\hline Insomnio Pre-Post & 0,484 & 0,91 & & & 0,172 & 0,81 & 0,10 & & 0,363 & 0,179 \\
\hline Funciones Intelectuales Pre-Post & 0,469 & 10,05 & 0,132 & 0,001 & 0,203 & 0,67 & 0,08 & 0,018 & 0,301 & 0,149 \\
\hline Humor Deprimido Pre-Post & 0,422 & 10,07 & 0,133 & 0,002 & 0,250 & 10,45 & 0,18 & 0,172 & 0,135 & 0,068 \\
\hline Síntomas Somáticos Generales Pre-Post & 0,516 & 10,084 & & 0,000 & 0,313 & 10,54 & 0,19 & 0,110 & 0,152 & 0,0759 \\
\hline Síntomas Somáticos Pre-Post & 0,563 & 10,11 & 0,139 & .000 & $-0,078$ & 10,60 & 0,20 & 0,697 & 0,466 & 0,227 \\
\hline Síntomas Cardiovascular Pre-Post & 0,391 & 0,88 & 0,111 & 0,001 & 0,000 & 10,60 & 0,20 & 10,000 & 0,302 & 0,149 \\
\hline Síntomas Respiratorios Pre-Post & 0,281 & 0,93 & 0,117 & 0,019 & $-0,141$ & 10,60 & 0,20 & 0,482 & 0,110 & 0,055 \\
\hline Síntomas Gastrointestinales Pre-Post & 0,438 & 10,14 & 0,142 & 0,003 & $-0,094$ & 10,38 & 0,17 & 0,588 & 0,272 & 0,135 \\
\hline Síntomas Genitourinarios Pre-Post & 0,141 & 0,587 & 0,073 & 0,060 & $-0,016$ & 10,686 & 0,21 & 0,941 & 0,099 & 0,050 \\
\hline Síntomas del SNC Autónomo Pre-Post & 0,453 & 10,04 & 0,130 & 0,001 & $-0,094$ & 10,60 & 0,20 & 0,641 & 0,266 & 0,132 \\
\hline
\end{tabular}

Las dimensiones con significación estadística $(p>0,05)$ en el grupo intervención fueron 12, Humor Ansioso, Tensión, Miedo, Insomnio, Funciones Intelectuales, Humor deprimido, Síntomas Somáticos Generales, Síntomas Somáticos, Síntomas Cardiovasculares, Síntomas Respiratorios, Síntomas Gastrointestinales y del SNC Autónomo. En tanto en el grupo testigo fueron dos dimensiones con significancia estadística; Humor Ansioso y Funciones Intelectuales.

En cuanto al tamaño del efecto, las dimensiones con tamaño pequeño $(0 \mathrm{a}$ $0,20)$ fueron cuatro: Humor Deprimido,

\section{DISCUSIÓN}

La presente investigación mide el nivel de ansiedad durante el periodo de hospitalización en el paciente pediátrico oncológico y su cuidador, dicha medición se llevó a cabo a través del CMAS-R y HAS respectivamente. Dentro de la literatura se señala la necesidad de llevar a cabo estudios de investigación de tipo cualitativo ante la importante escasez de estudios que apoyan el uso de musicoterapia ${ }^{(11)}$. De igual forma, cada vez más se está empleando la musicoterapia con rigurosidad científica y metodológica en la población de niños hospitalizados ${ }^{(4)}$. 
La hospitalización puede representar para el niño una situación que incrementa su propia vulnerabilidad, al encontrarse inmerso en una serie de estímulos estresantes $y$, posiblemente, amenazantes para su integridad física, lo cual genera alteraciones emocionales, cognitivas y de comportamiento $^{(12)}$. Por ello, es posible que los resultados obtenidos con los pacientes que participaron no muestren cambio significativo en el nivel total de ansiedad, pues los estímulos estresantes están presentes durante el tiempo de hospitalización, aunque sí hubo diferencia entre los niveles internos entre ausencia de ansiedad, ansiedad leve o ansiedad moderada o grave. En el caso del grupo intervención, a través de chi cuadrada señala que no hubo cambio en los niveles ausencia de ansiedad y ansiedad leve, mientras que en la post evaluación hubo aumento de cuatro a siete en el nivel moderado y de seis a nueve en el nivel grave, mientras que el nivel muy grave disminuyó de 33 a 27 . Este resultado coincide con otros informes de investigación en los que se emplea la música como recurso para el manejo de ansiedad, así lo reporta Yañez Amoros ${ }^{(13)}$, quién al hacer una revisión narrativa de diversos estudios con musicoterapia en pacientes oncológicos, en uno de los estudios se encontró que la terapia musical ayuda a reducir la ansiedad, a enfrentar los problemas físicos y aliviar las emociones que causan cáncer, según muestra las conclusiones de una investigación Ilevada a cabo en 2008 en el Centro Integral de Cáncer de la Universidad de Michigan (EEUU), donde el efecto de la musicoterapia sobre la ansiedad en el grupo de terapia fue significativamente inferior que el de los pacientes de control, demostrando la efectividad de la terapia para reducir la ansiedad. Para ello se utilizó el STAI-E-R, según lo reporta la autora. En nuestra medición, dicho cambio en el nivel de ansiedad no logra ser significativo, aunque concordamos con los datos de la literatura donde se asegura que la infancia es la fase de la vida en la que el ambiente influye más en el desarrollo. La música es capaz de estimular el desarrollo físico y cognitivo y fomenta la creatividad ${ }^{(14)}$, es un recurso globalizador en cuanto a que si se emplea adecuadamente pueden estar trabajándose al mismo tiempo objetivos de varias áreas de desarrollo ${ }^{(4)}$.

Ahora bien, en el grupo testigo la medición de los niveles internos de ansiedad en el paciente a través de chi cuadrada reflejó en la pre evaluación 15 pacientes con ausencia de ansiedad, siete con ansiedad leve, tres con ansiedad moderada, seis con ansiedad grave y 25 pacientes con nivel de ansiedad muy grave; mientras que en la post medición fueron 17 pacientes con ausencia de ansiedad, seis con ansiedad leve, siete con ansiedad moderada, cuatro con ansiedad grave y 22 con ansiedad muy grave. Por tanto, hubo aumento en el nivel de ausencia y moderada, mientras que el nivel leve, grave y muy grave disminuyó. Estos cambios aunque no hubo estímulo musical, es probable que la resiliencia del paciente oncológico influya positivamente de forma natural, o bien; que las actividades de terapia ocupacional que forman parte del cotidiano de actividades que se realizan en el Servicio de Hemato-oncología pediátrica, sean un factor que beneficie al paciente, por lo que es probable que se sienta menos ansioso $y$ menos sensible cuando se mantiene ocupado realizando alguna actividad, que es favorable mantenerlo ocupado mental y físicamente a través del juego creativo, recreativo e interactuando con el medio, ya sea con sus padres o el personal de salud que le asiste, tal vez una combinación entre música y juego, como se reportó en un estudio donde la mayoría de los participantes respondieron que es mejor trabajar con música, pues genera un ambiente alegre, calmado y sereno ${ }^{(14)}$. En tanto, el hallazgo en esta investigación puede in- 
cluso considerarse como una vertiente futura a indagar sobre la influencia del juego y ocupación del paciente oncológico en el periodo hospitalario.

Un aspecto que es por igual importante indagar, es la diferencia obtenida en la medición de ansiedad con relación al género, ya que se obtuvo cambio significativo sólo con el género femenino como se reportó en el apartado de resultados. En este sentido, el género puede ser uno de los factores que determinan las diferentes reacciones ante la experiencia de hospitalización que para unos puede ser traumática, mientras que para otros Ilega a ser adaptativa. Otro factor que es pertinente no olvidar, es que la hospitalización por naturaleza simboliza un evento que causa ansiedad, esto debido a las diversas amenazas que bien pueden ser reales -punciones, procedimientos dolorosos, vivencia física de la enfermedad-o imaginarias para el paciente pediátrico, lo que puede mantener niveles altos de ansiedad durante la estancia hospitalaria. De ello parte la importancia de atención psicooncológica oportuna, pues durante la fase de tratamiento el paciente requiere frecuentes periodos de hospitalización, lo que se transforma en estados recurrentes de ansiedad que puede Ilegar a ser de tipo anticipatoria cuando el paciente conoce la fecha para volver al hospital, o bien desencadenar trastornos de adaptación posteriores. De igual forma, es posible que la edad del paciente es una variante para mostrar factores estresantes que se vivencia de forma distinta. En este sentido, Ziegle y Prior (1994) especifican cinco rangos entre los que señalan que de los cero a los cinco años mostrarán ansiedad por separación y por lo desconocido, mientras que de los seis a los 18 años mostrarán miedo al dolor, a la mutilación, a la hospitalización a manera de castigo y a la muerte. Los adolescentes presentan miedo al rechazo de los amigos, a la pérdida de control y a la limitación de las actividades físicas así como a la pérdida de la independencia ${ }^{(15)}$. En el caso de la presente investigación una limitante es que el rango de edad de pacientes analizados fue amplio, por lo que no es posible contrastar lo propuesto por los autores con relación a la respuesta emocional a la hospitalización según la edad del paciente.

Por otro lado, dentro de la literatura, no se encontraron estudios con musicoterapia que se extiendan a la familia o al entorno del paciente con cáncer, por lo que es conveniente puntualizar la importancia de la interacción que se genera en su entorno, ya que el estado emocional, como bien puede ser el nivel de ansiedad, se convierte en un flujo entre paciente-cuidador por lo que ambos se retroalimentan constantemente. De igual forma, la hospitalización es un proceso en ocasiones nuevo que llega de forma inesperada en donde tanto el paciente como cuidador experimentan situaciones distintas a las que realizan en lo cotidiano, por lo que las reacciones emocionales adaptativas o estresantes deben ser esperadas en ambos. Las sesiones de musicoterapia brindan una experiencia a niños y familiares que ayuda a normalizar y familiarizarse con el ambiente hospitalario tan extraño y, en ocasiones, tan hostil ${ }^{(3)}$. Bajo este orden de ideas, se considera que durante la hospitalización el estado emocional en el cuidador es de importancia, ya que la confluencia antes mencionada entre paciente-cuidador puede ser direccionada para que sea favorable, una forma para hacerlo es a través de la musicoterapia, así lo demuestran los resultados obtenidos en esta investigación donde la pre evaluación del nivel de ansiedad en el grupo de cuidadores con intervención mostraron que ocho colaboradores presentaron ausencia de ansiedad, 20 ansiedad leve, 21 ansiedad moderada, 14 ansiedad grave y uno ansiedad muy grave. En tanto la post medición reflejó 20 colaboradores con ausencia de ansiedad, 26 con ansiedad leve, 12 con ansiedad moderada, cinco presentaron ansiedad grave y permaneció uno 
con ansiedad muy grave. Se observa entonces al escuchar la sesión musical un marcado aumento de colaboradores con ausencia de ansiedad y ansiedad leve, mientras que el nivel de ansiedad moderado y grave disminuyó significativamente. Ahora bien, los resultados de la medición del grupo testigo de cuidadores mantuvieron un equilibrio, la pre-evaluación reflejó 13 pacientes con ausencia de ansiedad y en la post medición aumentó a 17 colaboradores, la ansiedad leve, moderada y grave disminuyó de 16 a 15 , de 24 a 20 y de 11 a 10 respectivamente. Dos colaboradores mostraron nivel de ansiedad muy grave en la post-medición.

Un tema aparte mas no aislado de la relación antes planteada respecto a la edad y género como variantes de la respuesta al estímulo musical, es que los expertos en música señalan que existe gran desconocimiento de la biología que subyace o se relaciona con la musicoterapia, hay quienes señalan que es innata y que la corteza frontal tiene un papel importante en la percepción del ritmo y la melodía, otras investigaciones refieren que al escuchar música se presenta actividad en regiones del cerebro que controlan el movimiento. Aunque los expertos no coinciden en la forma en que responde el cerebro al estímulo musical, sí coinciden en que la respuesta cerebral depende de la experiencia y educación musical del oyente. Por ello, puede ser que la edad sea un factor importante a considerar si se desea emplear musicoterapia como recurso de apoyo psicoterapéutico, pues la infancia es el periodo donde se integran los sonidos y se presentan los primeros acercamientos con la música, ya sea de forma pasiva como oyente, o de manera activa con el aprendizaje lírico o dirigido de algún instrumento; mientras que en la edad adulta al existir previamente este registro en cualquiera de sus formas, la respuesta positiva ante la influencia musical es casi automática, pues existe mayor experiencia con la música, además de que hay mayor claridad en el gusto o preferencia musical.

\section{CONCLUSIONES}

Esta investigación permitió verificar cuantitativamente la ansiedad en el paciente pediátrico con cáncer y de su cuidador durante el periodo de hospitalización. El resultado de la comparación entre el grupo intervención y testigo lleva a la conclusión que en el paciente pediátrico oncológico no se presenta cambio significativo en el nivel de ansiedad empleando musicoterapia como recurso de apoyo, sin embargo; es importante considerar la edad, género, contexto hospitalario y sobre todo la vivencia física de la enfermedad como elementos que aunados a la respuesta emocional inciden de manera constante en el nivel de ansiedad. En el caso de los cuidadores que participaron en la investigación se observó evidente disminución en el nivel de ansiedad al escuchar la sesión musical, en tanto; en el grupo control aún sin la sesión musical hubo disminución de ansiedad aunque no logra ser tan significativo como en el grupo con intervención.

Finalmente, a partir de los resultados obtenidos concluimos que el uso de musicoterapia con los pacientes no logró una disminución de ansiedad de forma significativa, por lo que nuestros resultados no apoyan la idea generalizada del beneficio en pacientes pediátricos oncológicos. Sin embargo, consideramos que al obtener resultado favorable en la disminución del nivel de ansiedad en los cuidadores, se logra permear una influencia positiva al paciente a través de la transferencia emocional cuidador-paciente y que por lo tanto, influye en el modo en que se percibe la ansiedad durante el periodo de hospitalización, pues el cuidador es quien moralmente proporciona al paciente aspectos como protección, seguridad y compañía en gran medida. 


\section{REFERENCIAS BIBLIOGRÁFICAS}

1. Valdés CA, Flores JA. El niño ante el hospital, programa para reducir la ansiedad hospitalaria. Oviedo: Servicio de publicaciones de la Universidad de Oviedo; 1995.

2. May R. El surgimiento de la psicología existencial. Psicología Existencial. Buenos Aires: Gedisa, 1963.

3. MUSA Música y Salud [Internet]. Salamanca Herrero D, Pascual Toca R, Garrido Rosa N. Beneficios de la Musicoterapia en el Hospital Pediátrico. [Acceso el 15 de diciembre de 2012]. Disponible http://musicaysalud.org

4. Serradas Fonseca M. La música como medio de expresión del niño hospitalizado. Educere 2006;10:35-41.

5. Trallero Flix C. Musicoterapia creativa en pacientes con cáncer: un método para disminuir la ansiedad y el estrés. Revista virtual Psiquiatría.com [Internet]. 2004 [Acceso el 28 de mayo| de 2012]; 2:1-9. Disponible en: http://hdl.handle.net/2445/11516

6. Aldridge D. Music therapy references relating to cancer and palliative care. $\mathrm{Br}$ J Music Ther 2003;17: 17-25.

7. Miquelarena M. El cuerpo y la música en el tratamiento psiquiátrico. Editorial Medina Montevideo: Uruguay, 1987.

8. Ortíz González AL. Ansiedad y miedos en niños ante la hospitalización. Investigación, intervención, programas y técnicas. Revista de la Facultad de Psicología Universidad Cooperativa de Colombia 2006;3: 84100. [Acceso 10 de septiembre de 2013]. Disponible en: http://wb.ucc.edu.co/ pensandopsicologia/files/2010/09/articulo11-vol3-n3.pdf

9. Reynolds $\mathrm{Cr}$, Richmond BO. Escala de ansiedad manifiesta en niños (revisada) CMAS-R. México: Manual Moderno, 1997.

10. Carrobles JA, Costa M, Del SerT, Bartolomé P. La práctica de la terapia conductual. Valencia: Promolibro, 1986.

11. Russell EH. Musicoterapia en residencias para enfermos terminales y cuidados paliativos: revisión de datos empíricos. Music Department, Sate University of New York. 2005;2 (2):173-8.

12. Fernández C, López N. Transmission of emotions, fear, and pediatric strees in hospitalization. Int j Clin Health Psychol 2006;6 (3):631-645.

13. Yañez Amoros B. Musicoterapia en el paciente oncológico. CultCuid 2011;15:5773. Doi:10.7184/cuid.2011.29.07

14. Correa I, Guedelha BD. Utilización de la música en busca de la asistencia humanizada en el hospital. Invest Educ Enferm 2009;27:46-50.

15. Ziegler DB, Prior M. Preparation for surgery and adjustment to hospitalization. Pediatric Surgical Nursing 1994;29 (4):655-69. 


\section{ANEXO}

\section{CRITERIOS GENERALES DE SELECCIÓN MUSICAL}

El material musical seleccionado para las sesiones con el grupo intervención de pacientes y cuidadores consistió en un total de 6 melodías que conformaron el repertorio de música en los periodos barroco, clásico, romántico, impresionista, moderno, así como música folclórica de diferentes países como Brasil, Venezuela, Cuba, España e Italia. La selección de las obras musicales fue realizada con la participación profesional de una psicóloga experta en música y se llevó a cabo una prueba piloto que permitió hacer ajustes sobre todo en el tiempo de la sesión.

Para las sesiones musicales se seleccionaron diferentes rítmicas, timbres de instrumentos y formas melódicas ya que estas son recibidas por el participante -oyentede diferente manera según la percepción y antecedente respecto a su acercamiento con la música. Así, las sesiones musicales estuvieron compuestas por seis melodías derivadas de tres grandes vertientes: música clásica con orquesta, renacentista/ medieval con instrumento musical y folclórica. Las obras musicales que sirvieron de estímulo experimental para el manejo de ansiedad, cumplieron los siguientes requisitos:

a) Música con tonos afectivos: tonalidad mayor, que según la Teoría Modal de los griegos, esta música sugiere apertura, alegría, determinación.

b) Música instrumental: para facilitar la recepción y percepción sólo del estímulo musical

c) Melodías claras para evitar distractores o que fueran complejas incluso para ser sólo escuchadas. Se elige la música clásica por la variedad de timbres y la gama de instrumentos que conforman la orquesta, como las cuerdas, alientos, metales y percusiones.

d) Música de diferentes épocas y estilos: al considerar música de otras culturas, con diferencia en su tonalidad, ritmo e instrumentos generan estados mentales a los que no estamos acostumbrados y que por tanto, evocan alguna particularidad en el estado mental y consciente del ser humano. la música de corte antiguo consta de instrumentos poco conocidos por ser propios de la edad media como violas de gamba, clavecines, chirimías y laudes. Finalmente, la música folclórica tradicional incluyó ritmos de diversos países, ya que el factor común que comparten es el ritmo en forma de danza.

Las melodías se escucharon de manera aleatoria, esto con la finalidad de separar los ritmos para que los participantes pudieran diferenciar las melodías, siendo el orden el siguiente: música clásica con orquesta, música teniendo como solista un instrumento, música folclórica tradicional, música clásica con orquesta, música medieval/renacentista y una última melodía de música folclórica tradicional. El tiempo de escucha determinado para cada melodía fueron tres minutos, esto debido a que se consideró tiempo suficiente para que la vibración de los sonidos y tonos de las melodías generaran un impacto en el estado anímico, para obtener piezas con esta duración, se delimitó el tiempo inicial de cada melodía, algunas fueron editadas para eliminar puentes, enlaces o partes repetidas. Al final de cada melodía, el participante tuvo oportunidad de calificarla de acuerdo a su gusto o preferencia musical a través de una escala Likert. 
Se presenta a continuación la cita de las 6 obras musicales correspondientes a cada sesión realizada, se encontrarán en el mismo orden que fueron reproducidas.

\begin{tabular}{|c|c|c|c|c|}
\hline SESIÓN & MELODÍA & AUTOR & INTERPRETADO POR & GÉNERO MUSICAL \\
\hline \multirow{6}{*}{1} & $\begin{array}{l}\text { Las cuatro estaciones. La } \\
\text { primavera }\end{array}$ & Antonio Vivaldi & $\begin{array}{l}\text { Orquesta Sinfónica de la } \\
\text { Sociedad Filarmónica de } \\
\text { conciertos (México) }\end{array}$ & Música clásica de orquesta \\
\hline & La Noyee & Yan Tiersen & Yan Tiersen & Instrumento: Piano \\
\hline & O pato & Jayme Silvia & Tempo rey & $\begin{array}{l}\text { Música Folclórica Tradicional, } \\
\text { Brasil }\end{array}$ \\
\hline & $\begin{array}{l}\text { Concierto en D mayor } \\
\text { op. } 35\end{array}$ & Piotr Ilich Tchaikovsky & Sayaka Sojhi & Música clásica de orquesta \\
\hline & Dindirin, Dindirin & Anónimo & Carles Magraner & Música medieval \\
\hline & El Querreque & $\begin{array}{l}\text { Arreglo del Sr. Willebaldo } \\
\text { Amador Hernández }\end{array}$ & Grupo Chicontepee & $\begin{array}{l}\text { Música Folclórica Tradicional } \\
\text { México }\end{array}$ \\
\hline \multirow{6}{*}{2} & Ronda alla Turca & $\begin{array}{l}\text { Wolfgang Amadeus } \\
\text { Mozart }\end{array}$ & Mitzuko Uchida & Música clásica de orquesta \\
\hline & Por ti Volare & $\begin{array}{l}\text { Lucio Quarantotto, } \\
\text { Francesco Sartori y Luis } \\
\text { Gómez Escolar }\end{array}$ & Andrea Bocelli & Instrumento: $V_{0 z}$ \\
\hline & Casatschok Loy Loy & Mijail Isakowsky & Cosak Rusian Gregorian Dance & Música clásica de orquesta \\
\hline & Huapango de Moncayo & José Pablo Moncayo & Filarmónica de Berlín & $\begin{array}{l}\text { Música Folclórica Tradicional, } \\
\text { México }\end{array}$ \\
\hline & Canon in D Major & Johan Christoph Pachelbel & David and the high spirit & Música Renacimiento/barroco \\
\hline & $\begin{array}{l}\text { Lo mejor de la música } \\
\text { Ilanera }\end{array}$ & Hipólito Arrieta & Música llanera & $\begin{array}{l}\text { Música Folclórica Tradicional, } \\
\text { Venezuela }\end{array}$ \\
\hline \multirow{6}{*}{3} & Concerto in D Major RV 558 & Antonio Lucio Vivaldi & Giovanni Antonini & Música clásica de orquesta \\
\hline & The devil's Trill & Guisepe Tartini & Vanesa Mae & Instrumento: Violín \\
\hline & Carnaval & Sergio Santos Mendez & Sergio Santos Mendez & \begin{tabular}{|l|} 
Música Folclórica Tradicional, \\
Brasil \\
\end{tabular} \\
\hline & Sinfonía 40 Molto Allegro & Wolfang Amadeus Mozart & Filarmónica de Viena & Música Clásica de Orquesta \\
\hline & Cantinga 166 & Alfonso X el Sabio & Vox Vulgaris & Música Medieval \\
\hline & El Son de la Negra & Blas Galindo & Mariachi Vargas de Tecalitlán & $\begin{array}{l}\text { Música Folclórica Tradicional, } \\
\text { México }\end{array}$ \\
\hline \multirow{6}{*}{4} & $\begin{array}{l}\text { Sinfonía No. } 9 \\
\text { (Himno de la Alegría) }\end{array}$ & Ludwig van Beethoven & John Marshall & Música Clásica de Orquesta \\
\hline & Corazón de Niño & Raúl Di Blasio & Raúl Di Blasio & Instrumento: Piano \\
\hline & El carretero & Ronald Rubinel & Grupo Putumayo & $\begin{array}{l}\text { Música Folclórica Tradicional, } \\
\text { Cuba }\end{array}$ \\
\hline & Gloria RV 589 & Antonio Vivaldi & $\begin{array}{l}\text { The Choir of Chirst Church } \\
\text { Cathedral Oxford } \\
\end{array}$ & Música Clásica de Orquesta \\
\hline & Chacona a la vida bona & Juan Arañés & La Capella de Catalunya & Música Renacentista \\
\hline & El Cielito Lindo & Quirino Mendoza y Cortez & Los camperos del Valle & $\begin{array}{l}\text { Música Folclórica Tradicional, } \\
\text { México }\end{array}$ \\
\hline \multirow{6}{*}{5} & Las bodas de Figaro & Wolfgang Amadeus Mozart & $\begin{array}{l}\begin{array}{l}\text { Orquesta Filarmónica de } \\
\text { Londres }\end{array} \\
\end{array}$ & Música Clásica de Orquesta \\
\hline & Mandolin Concerti & Antonio Lucio Vivaldi & Claudio Scimone, Dorina Frati & Instrumento: Mandolín \\
\hline & \begin{tabular}{|l|} 
Fandango y Jarana/La \\
Marimorena
\end{tabular} & A.R, Ferrera/Anónimo & Grupo Tierra de Barros & $\begin{array}{l}\text { Música Folclórica Tradicional, } \\
\text { España }\end{array}$ \\
\hline & Russischer Marsch Op. 426 & Johan Strauss Sohn & Filarmónica de Viena & Música clásica de Orquesta \\
\hline & La Sol Fa Mi Re & Gaspar Fernández & Ars Nova & Música Virreinal Mexicana \\
\hline & El tilingo lingo & Lino Carillo & Grupo Chucumite & $\begin{array}{l}\text { Música Folclórica Tradicional, } \\
\text { México }\end{array}$ \\
\hline
\end{tabular}




\begin{tabular}{|c|c|c|c|c|}
\hline SESIÓN & MELODÍA & AUTOR & INTERPRETADO POR & GÉNERO MUSICAL \\
\hline \multirow{6}{*}{6} & Vals de las flores & Piotr Ilich Tchaikovsky & South China Music Troup & Música Clásica de Orquesta \\
\hline & Arts of War & Vanessa Mae & Vanessa Mae & Instrumento: Violín \\
\hline & Jarabe Tapatío & $\begin{array}{l}\text { José Leopoldo Enrique } \\
\text { Reyes Oliva }\end{array}$ & Mariacho Vargas de Tecalitlán & $\begin{array}{l}\text { Música Folclórica Tradicional, } \\
\text { México }\end{array}$ \\
\hline & $\begin{array}{l}\text { Concierto para piano in D } \\
\text { Major No. } 21\end{array}$ & Wolfgang Amadeus Mozart & Alfonso Gómez & Música clásica de Orquesta \\
\hline & A este edificio célebre & $\begin{array}{l}\text { Sor Juana Inés de la Cruz, } \\
\text { música de Andrés Flores }\end{array}$ & Cantores de Catalunya & Música Medieval \\
\hline & Pájaro Chogüi Hecatl & Guillermo Breer & $\begin{array}{l}\text { Grupo Andino de la } \\
\text { Universidad de Sotavento }\end{array}$ & $\begin{array}{l}\text { Música Folclórica Tradicional } \\
\text { Andina }\end{array}$ \\
\hline \multirow{6}{*}{7} & $\begin{array}{l}\text { Concierto para clarinete en } \\
\text { A 1st allegro }\end{array}$ & Wolfgang Amadeus Mozart & $\begin{array}{l}\text { Sharon Kam, Czech } \\
\text { Philarmonic }\end{array}$ & Música clásica de orquesta \\
\hline & Mission Imposible & Borris Claudio Schifrin & $\begin{array}{l}\text { Orquesta Sinfónica. Piano } \\
\text { Schifrin } \\
\text { Trompeta James Morrison }\end{array}$ & Instrumento: trompeta y piano \\
\hline & Tarantella Siciliana & Renzo Arbore & Grupo Folk Naxos & $\begin{array}{l}\text { Música Folclórica Tradicional, } \\
\text { Sicilia }\end{array}$ \\
\hline & Rosas del sur & Johann Strauss II & Andre Rieu & Música clásica Orquesta \\
\hline & Excalibur el "Tourdion" & Ritchie Blackmore & Blackmore Knights & Música renacentista \\
\hline & Moliendo café & Jose Manso Perroni & Los Andes & $\begin{array}{l}\text { Música Folclórica tradicional } \\
\text { andina }\end{array}$ \\
\hline \multirow{6}{*}{8} & $\begin{array}{l}\text { Pequeña serenata nocturna- } \\
\text { Allegro }\end{array}$ & Wolfgang Amadeus Mozart & $\begin{array}{l}\text { Florian Heyerick y } \\
\text { Hurpfalzisches }\end{array}$ & Música Clásica Orquesta \\
\hline & Dance Macabre 2010 & Camille Saint-Saëns & $\begin{array}{l}\text { Orquesta de París Piano: } \\
\text { Daniel Barenboim } \\
\text { Violín: Luben Yordanoff }\end{array}$ & Instrumento: piano y violín \\
\hline & $\begin{array}{l}\text { Música Oaxaqueña } \\
\text { "Jarabe Mixteco" }\end{array}$ & $\begin{array}{l}\text { Francisco Cipriano Villa } \\
\text { Hernández y Antonio } \\
\text { Martínez Corro } \\
\end{array}$ & $\begin{array}{l}\text { Banda de músicos del Estado } \\
\text { de Oaxaca }\end{array}$ & $\begin{array}{l}\text { Música Folclórica Tradicional, } \\
\text { México }\end{array}$ \\
\hline & Danza Bachanelle & Camille Saint-Saëns & Orquesta Juvenil de Caracas & Música Clásica de Orquesta \\
\hline & Wonderful Medieval music & Azam Alí & Ensamble Anonymus & Música medieval \\
\hline & El son de la loma & Miguel Matamoros & Trío Caribe & $\begin{array}{l}\text { Música Folclórica Tradicional, } \\
\text { Cuba }\end{array}$ \\
\hline \multirow{6}{*}{9} & $\begin{array}{l}\text { Divertimento in D major } \\
k 136\end{array}$ & Wolfgang Amadeus Mozart & Chamber Orchestra & Música Clásica Orquesta \\
\hline & $\begin{array}{l}\text { Prelude and Fugue no. } 1 \text { in } \\
\text { C major BWV } 846\end{array}$ & Johann Sebastian Bach & Kenneth Gilbert & Instrumento: Clave \\
\hline & La culebra & Ruben Fuentes & Mariachi Vargas de Tecalitlán & $\begin{array}{l}\text { Música Folclórica Tradicional, } \\
\text { México }\end{array}$ \\
\hline & $\begin{array}{l}\text { Haydn cello concerto in } \\
\text { D part. } 1\end{array}$ & Joseph Haydn & Misha Maiskyjor & Música Clásica Orquesta \\
\hline & $\begin{array}{l}\text { Instrumental dance } \\
\text { Frederick II'court }\end{array}$ & Sebastiano Occhino & $\begin{array}{l}\text { Banda Sonora Musique de } \\
\text { dance medievale }\end{array}$ & Música Medieval \\
\hline & El cuculi de la flauta & Joel Francisco Perri & Joel Francisco Perri & $\begin{array}{l}\text { Música Folclórica Tradicional, } \\
\text { Perú }\end{array}$ \\
\hline \multirow{6}{*}{10} & $\begin{array}{l}\text { Symphony No. } 22 \text { in C } \\
\text { Major KV } 162 \text { Allegro Assai }\end{array}$ & Wolfgang Amadeus Mozart & Northern Chamber Orquesrta & Música Clásica Orquesta \\
\hline & Radinerie & Johan Sebastian Bach & James Galway & Instrumento: Flauta \\
\hline & Música Tradicional China & Anónimo & Anónimo & $\begin{array}{l}\text { Música Folclórica Tradicional, } \\
\text { China }\end{array}$ \\
\hline & Symphony No.1 in E flat major & Wolfgang Amadeus Mozart & Trevor Pinnock & Música Clásica Orquesta \\
\hline & Sahara & Cultus Ferox & Cultus Ferox & Música Medieval \\
\hline & Pájaro campana & Anónimo & Manuel Ángel López & $\begin{array}{l}\text { Música Folclórica Tradicional, } \\
\text { Paraguay }\end{array}$ \\
\hline
\end{tabular}




\begin{tabular}{|c|c|c|c|c|}
\hline SESIÓN & MELODÍA & AUTOR & INTERPRETADO POR & GÉNERO MUSICAL \\
\hline \multirow{6}{*}{11} & $\begin{array}{l}\text { Concierto en Do Mayor } \\
\text { para } 2 \text { Trompetas Mov. } 1\end{array}$ & Antonio Vivaldi & $\begin{array}{l}\text { Orquesta José Perpiñon de } \\
\text { Sergobe, solistas Ramón Caro y } \\
\text { Sergio Campos }\end{array}$ & Música Clásica Orquesta \\
\hline & Sonata in A Mayor K.24 & Domenico Scarlatti & Igor Kipnis & Instrumento: Harpsichord \\
\hline & Abre o fole tio bilia & Renato Borghettio & $\begin{array}{l}\text { Los Serranos y Renato } \\
\text { Borghettio }\end{array}$ & $\begin{array}{l}\text { Música Folclórica Tradicional, } \\
\text { Brasil }\end{array}$ \\
\hline & $\begin{array}{l}\text { Concerto para flauta en sol } \\
\text { Major kv. } 3131\end{array}$ & Wolfgang Amadeus Mozart & Cesar Peredo & Música Clásica Orquesta \\
\hline & Medieval Folks & & & Música medieval \\
\hline & Federico Boogaloo & Federico Junior & Sonora Danceliz & $\begin{array}{l}\text { Música Folclórica Tradicional, } \\
\text { Colombia }\end{array}$ \\
\hline \multirow{6}{*}{12} & Sleeping Beauty Walts & Piotr Ilich Tchaikovsky & $\begin{array}{l}\text { New Festival Orquestra \& } \\
\text { Moshe Nussbaum }\end{array}$ & Música Clásica Orquesta \\
\hline & Caprice No. 24 & Nicolo Paganini & Jasmine Choi & Instrumento: Flauta \\
\hline & La Leva & Elpidio Ramírez & Trío Guardianes de la Huasteca & $\begin{array}{l}\text { Música Folclórica Tradicional, } \\
\text { México }\end{array}$ \\
\hline & $\begin{array}{l}\text { Sinfonía de los juguetes } \\
\text { Allegro }\end{array}$ & Leopoldo Mozart & $\begin{array}{l}\text { Orquesta Sinfónica } \\
\text { Universidad de Pereira }\end{array}$ & Música Clásica Orquesta \\
\hline & $\begin{array}{l}\text { Danza la brosse, Danza la } \\
\text { Gatta, Danza Mon Desir }\end{array}$ & Pierre Attaignant & $\begin{array}{l}\text { Josef Ulsamer y Collegium } \\
\text { Ulsamer }\end{array}$ & Música Medieval \\
\hline & Ran Kan Kan/oye como va & Tito Puente & Dave Valentin, Ray Vega & $\begin{array}{l}\text { Música Folclórica Tradicional, } \\
\text { Puerto Rico }\end{array}$ \\
\hline \multirow{6}{*}{13} & $\begin{array}{l}\text { Flaute concerto in D Major } \\
\text { K.314 Allegro aperto } \\
\end{array}$ & Wolfgang Amadeus Mozart & $\begin{array}{l}\text { Flauta: Ayda Chan } \\
\text { Piano: Paul Switzler }\end{array}$ & Música Clásica Orquesta \\
\hline & Red Hot 720 & Vanesa Mae & Vanessa Mae & Instrumento: Violín \\
\hline & El Puntalito & Constantino Blanco Ruíz & $\begin{array}{l}\text { Miguel Romero, Graciana } \\
\text { Silva, Felipe Ochoa }\end{array}$ & $\begin{array}{l}\text { Música Folclórica Tradicional, } \\
\text { México }\end{array}$ \\
\hline & $\begin{array}{l}\text { Oboe Concerto in C K.314 } \\
\text { I Allegro Aperto }\end{array}$ & Wolfgang Amadeus Mozart & $\begin{array}{l}\text { Heinz Holliger (oboe) } \\
\text { Orquesta de Chambre de } \\
\text { Lausanne }\end{array}$ & Música Clásica Orquesta \\
\hline & Sefardí 3/3 Sarband & Anónimo & Sarband & Música medieval \\
\hline & Vírgenes del sol & Jorge Bravo de rueda & Alejandro Vivanco & $\begin{array}{l}\text { Música Folclórica Tradicional } \\
\text { andina }\end{array}$ \\
\hline
\end{tabular}

